\title{
Minimal neutral naturalness model
}

\author{
Ling-Xiao $\mathrm{Xu},{ }^{1, \dagger}$ Jiang-Hao Yu $\odot,{ }^{2,3, *}$ and Shou-hua Zhu ${ }^{1,4,5, *}$ \\ ${ }^{1}$ Department of Physics and State Key Laboratory of Nuclear Physics and Technology, Peking University, \\ Beijing 100871, China \\ ${ }^{2}$ CAS Key Laboratory of Theoretical Physics, Institute of Theoretical Physics, \\ Chinese Academy of Sciences, Beijing 100190, People's Republic ofChina \\ ${ }^{3}$ School of Physical Sciences, University of Chinese Academy of Sciences, \\ No. 19A Yuquan Road, Beijing 100049, People's Republic of China \\ ${ }^{4}$ Collaborative Innovation Center of Quantum Matter, Beijing 100871, China \\ ${ }^{5}$ Center for High Energy Physics, Peking University, Beijing 100871, China
}

(Received 20 October 2018; accepted 1 May 2020; published 13 May 2020)

\begin{abstract}
We build a minimal neutral naturalness model in which the top partners are not charged under QCD, with a pseudo Nambu-Goldstone Higgs arising from $S O(5) / S O(4)$ breaking. The color-neutral top partners generate the Higgs potential radiatively without quadratic divergence. The misalignment between the electroweak scale and global symmetry breaking scale is naturally obtained from suppression of the Higgs quadratic term, due to cancellation between singlet and doublet top partner contributions. This model can be embedded into ultraviolet holographic setup in composite Higgs framework, which even realizes finite Higgs potential.
\end{abstract}

DOI: 10.1103/PhysRevD.101.095014

\section{INTRODUCTION}

The hierarchy problem remains as one of the unsolved puzzles in the Standard Model (SM), i.e., the Higgs mass is sensitive to the Planck scale through quantum effects. Symmetry for the Higgs boson, such as supersymmetry and shift symmetry, is typically introduced to relate the top quark with top partner, which lowers sensitivity of the ultraviolet (UV) scale in the Higgs mass down to the top partner scale. However, current experimental searches of the colored top partners have already set the lower bound of their masses around $1 \mathrm{TeV}$ [1-4], which leads to the little hierarchy problem [5].

One novel solution to the little hierarchy problem is a neutral naturalness scenario [6-19], in which top partners are not charged under the SM color group. The current search limit on masses of the colorless top partners is still below $\mathrm{TeV}[20,21]$, which softens the little hierarchy problem. Twin Higgs is the first example of neutral naturalness, in which the Higgs boson is identified as a

\footnotetext{
${ }^{*}$ Corresponding author. jhyu@itp.ac.cn

${ }^{\dagger}$ lingxiaoxu@pku.edu.cn

*shzhu@pku.edu.cn
}

Published by the American Physical Society under the terms of the Creative Commons Attribution 4.0 International license. Further distribution of this work must maintain attribution to the author(s) and the published article's title, journal citation, and DOI. Funded by SCOAP ${ }^{3}$. pseudo Nambu-Goldstone boson (PNGB) due to an accidental $Z_{2}$ between the SM and its twin copy. Although this idea is conceptually simple, it introduces many particles in the hidden sector, i.e., mirror $W^{\prime}, Z^{\prime}, \gamma^{\prime}$, and a whole generation of chiral fermions for anomaly cancellation. As a result, this setup for the hidden sector suffers from cosmological constraints due to the presence of hidden neutrinos and hidden photons [22-25]. It is well motivated to find alternative constructions for more minimal hidden sectors: mirror copies of the SM gauge bosons are not necessary, vectorlike fermions instead of chiral ones are introduced, i.e., no need for the whole generation of fermions.

In this paper, we present concrete neutral naturalness models with the minimal hidden sector. We only introduce the dark $S U(3)_{c}^{\prime}$ gauge symmetry in the hidden sector and adopt the minimal $S O(5) / S O(4)$ coset [26] for the PNGB Higgs. We also introduce minimal numbers of elementary vectorlike fermions: one singlet and one doublet of $S U(2)_{L}$, for canceling quadratic divergence from the top quark contribution, and realizing vacuum misalignment [27] between the electroweak scale $v$ and the global symmetry breaking scale $f$, i.e., $v \ll f$. We denote our model as the minimal neutral naturalness model (MNNM). As we will see, by our assignment of embeddings of top partners into representation of the $S O(5)$ symmetry, vacuum misalignment is naturally realized by cancellation between the contributions from different top partners. The potential of the PNGB Higgs can generally be parametrized $[28,29]$ as 


$$
V(h) \simeq-\gamma \sin ^{2}\left(\frac{h}{f}\right)+\beta \sin ^{4}\left(\frac{h}{f}\right),
$$

where $\gamma$ and $\beta$ denote radiative corrections from gauge boson and fermion contributions, and the electroweak scale is obtained by $\xi \equiv \frac{v^{2}}{f^{2}}=\frac{\gamma}{2 \beta}$. Radiative Higgs potential typically gives $\gamma \simeq \beta$ and thus $v \simeq f$. To realize vacuum misalignment $v \ll f$, one needs to either suppress the value of $\gamma$ or increase $\beta$. In conventional models, vacuum misalignment is usually realized between tuning of two independent sectors, e.g., $\gamma$ can be suppressed by cancellation between fermion and gauge boson contributions in twin Higgs [30,31]. In contrast, we show in this work for the first time this suppression of $\gamma$ can happen within one sector, i.e., the top quark sector, due to cancellation between the SM top quark and the color-neutral top partners, as fermions with different quantum numbers have different Higgs dependence.

In traditional neutral naturalness models, including the twin Higgs setup based on coset $S U(4) / S U(3)$ [8] (or $S O(8) / S O(7)$ [12-14] if custodial symmetry is incorporated) and the model with $S O(6) / S O(5)[15,16]$, the Higgs potential generated by the top sector respects the parity $s_{h} \leftrightarrow c_{h}$, and thus $\xi=1 / 2$. Typically additional paritybreaking terms are responsible for adjusting the value of $\xi$ from $\xi=1 / 2$ to phenomenologically viable ones, such as $\xi=0.1$. As we will show, it turns out fermion embedding in the $S O(5) / S O(4)$ model is different from all the above cosets. As a result, the small misalignment angle $\xi$ comes from exactly the same Lagrangian of top partners that is responsible for cutting off the quadratic divergence in the top sector. This motivates us to construct a neutral naturalness model with the $S O(5) / S O(4)$ coset, which can accommodate small $\xi$ without additional unknown sources that break the $s_{h} \leftrightarrow c_{h}$ parity.

To have our setup valid at UV scale, we extend MNNM by including composite states in the holographic framework [32]. Following spirit of composite Higgs models [12-17,26,33-35], we present a holographic MNNM and its deconstructed version. This brings finiteness of the Higgs potential, i.e., not sensitive, to the UV cutoff. After integrating out composite states, we recover the MNNM spectrum.

\section{THE MODEL}

Let us first introduce the field content of the hidden sector. The SM gauge symmetry is extended to $S U(3)_{c}^{\prime} \times$ $S U(3)_{c} \times S U(2)_{L} \times U(1)_{Y}$ where $S U(3)_{c}^{\prime}$ is an unbroken dark color gauge group. We introduce two vectorlike fermions $\tilde{q} \equiv(\tilde{t}, \tilde{b})^{T}$ and $\tilde{T}$, which are QCD neutral but carry the dark QCD charge,

$$
\tilde{q} \sim(3,1,2)_{Y}, \quad \tilde{T} \sim(3,1,1)_{Y},
$$

under $S U(3)_{c}^{\prime} \times S U(3)_{c} \times S U(2)_{L} \times U(1)_{Y}$, with $Y$ arbitrarily chosen. Introducing only the singlet $\tilde{T}$ can cancel the quadratic divergence as the top partner. However, to obtain the realistic Higgs potential with vacuum misalignment, here the additional doublet $\tilde{q}$ is introduced.

All the fermion contents are embedded into representations of global symmetry, in which the Higgs is a PNGB from global symmetry breaking. We adopt the minimal coset $S O(5) / S O(4)$ [26] incorporating the custodial symmetry in the Higgs sector, but do not include any compositeness from strong dynamics. The Higgs boson is represented by the nonlinear sigma field in unitary gauge as $\Sigma=f\left(0,0,0, s_{h}, c_{h}\right)^{T}$, where $s_{h} \equiv \sin (h / f), \quad c_{h} \equiv$ $\cos (h / f)$. The SM $S U(2)_{L} \times U(1)_{Y}$ symmetry is embedded in $S O(4) \times U(1)_{X} \cong S U(2)_{L} \times S U(2)_{R} \times U(1)_{X}$ with the hypercharge $Y=X+T_{R}^{3}$. The additional $U(1)_{X}$ is needed to obtain the correct hypercharge for the SM quarks. The SM doublet $q_{L}=\left(t_{L}, b_{L}\right)^{T}$ and singlet $t_{R}$ are embedded into 5-plet and singlet of the $S O(5)$, respectively,

$$
Q_{L}=\frac{1}{\sqrt{2}}\left(\begin{array}{c}
b_{L} \\
-i b_{L} \\
t_{L} \\
i t_{L} \\
0
\end{array}\right) \subset \mathbf{5}, \quad t_{R} \subset \mathbf{1}
$$

with quantum number $X=2 / 3$. In the hidden sector, the vectorlike fermions $\tilde{q}_{L, R}$ and $\tilde{T}_{L, R}$ are embedded as follows:

$\tilde{Q}_{L}=\frac{1}{\sqrt{2}}\left(\begin{array}{c}\tilde{b}_{L} \\ -i \tilde{b}_{L} \\ \tilde{t}_{L} \\ i \tilde{t}_{L} \\ \sqrt{2} \tilde{T}_{L}\end{array}\right) \subset \mathbf{5}, \quad \tilde{Q}_{R}=\frac{1}{\sqrt{2}}\left(\begin{array}{c}\tilde{b}_{R} \\ -i \tilde{b}_{R} \\ \tilde{t}_{R} \\ i \tilde{t}_{R} \\ 0\end{array}\right) \subset \mathbf{5}$,

$\tilde{T}_{R} \subset \mathbf{1}$,

with the same $U(1)_{X}$ charge. Once a mirror parity exchanging the SM top and color-neutral top partners is imposed, the $U(1)_{X}$ charge of the top partners should be the same as the SM top quark. As we will see later, this point is crucial to guarantee the cutoff scale is the same in the SM top sector and color-neutral top-partner sector, as the cutoff scale is interpreted as the scale of composite states in these two sectors, respectively. With the same $U(1)_{X}$ charge, one can define the same composite scale in these two sectors.

After embedding all the above elementary fermions in $S O(5)$ representations, we write down the following Lagrangian for the top Yukawa sector:

$$
\mathcal{L}_{\text {top }}=-y \bar{Q}_{L} \Sigma t_{R}-\tilde{y} \overline{\tilde{Q}}_{L} \Sigma \tilde{T}_{R}-m_{\tilde{q}} \overline{\tilde{Q}}_{L} \tilde{Q}_{R}+\text { H.c. }
$$


The last term is the mass term for the vectorlike doublet, which is allowed by the $S O(5)$ global symmetry. There is no mixing between the SM top quark and the top partners as they carry different $S U(3)_{c}^{\prime} \times S U(3)_{c}$ charges. The $\tilde{y} \overline{\tilde{Q}}_{L} \Sigma \tilde{T}_{R}$ term causes mixing between the hidden singlet and doublet top partners, with the mixing angle approximately $\tilde{y} f s_{h} / m_{\tilde{q}}$. The doublet fermion decouples when $m_{\tilde{q}} \rightarrow \infty$.

In order to cancel quadratic divergence from the top quark loop, symmetries need to be imposed in the top Yukawa terms in Eq. (5). Here we adopt the global $S U(6)_{c}$ symmetry in the Yukawa sector with $S U(3)_{c}$ and $S U(3)_{c}^{\prime}$ gauged: $S U(3)_{c} \times S U(3)_{c}^{\prime} \subset S U(6)_{c}$. Under the global symmetry $S O(5) \times S U(6)_{c}$, we define the bifundamental fermions based on Eqs. (3) and (4):

$$
\mathcal{Q}_{L}=\left(Q_{L}, \tilde{Q}_{L}\right), \quad \mathcal{T}_{R}=\left(t_{R}, \tilde{T}_{R}\right)
$$

The Yukawa terms in Eq. (5) are rewritten as

$$
\mathcal{L}_{\text {top }} \supset y \overline{\mathcal{Q}}_{L} \Sigma \mathcal{T}_{R}+\text { H.c. }, \quad \text { with } \quad y=\tilde{y} .
$$

Alternatively, introducing a $Z_{2}$ symmetry, which is external to $S O(5)$, in Eq. (5) between two $S O(5)$ multiplets

$$
Z_{2}: Q_{L} \leftrightarrow \tilde{Q}_{L}, \quad t_{R} \leftrightarrow \tilde{T}_{R},
$$

which indicates $y \bar{Q}_{L} \Sigma t_{R} \leftrightarrow \tilde{y} \overline{\tilde{Q}}_{L} \Sigma \tilde{T}_{R}$, and leads to quadratic divergence cancellation with $y=\tilde{y}$. In order to have a neutral-naturalness cancellation, we also introduce an internal-SO (5) rotational symmetry, which leads to the embeddings of singlet and doublet top partners in the $\tilde{Q}_{L}$ with appropriate coefficients, as shown in Eq. (4). This $S O(5)$ symmetry is fully elaborated in the Appendix. In brief, $\tilde{Q}_{L}$ is completed into a full $S O(5)$ 5-plet by introducing additional spurious fermions, and its different components are related by $S O(5)$ rotations. The parity defined as in Eq. (8) is uplifted to relate the full $S O(5)$ 5-plets. Different from the $Z_{2}$ parity [8] or the internal trigonometric parity [16] from outer automorphism, in which $t_{L} \leftrightarrow \tilde{t}_{L}, t_{R} \leftrightarrow \tilde{t}_{R}, c_{h} \leftrightarrow s_{h}$ at the particle level, the $Z_{2}$ symmetry introduced here is only respected between two $S O(5)$ fundamental representations. The advantage of imposing such symmetry at the Lagrangian level is that there is no need to introduce additional softly breaking terms to realize the correct vacuum misalignment, because we do not implement the $Z_{2}$ symmetry at the particle level. In contrast, additional $Z_{2}$ breaking sources are necessary in conventional twin Higgs setups with trigonometric parity; otherwise the Higgs potential would be $s_{h} \leftrightarrow c_{h}$ symmetric. In the Appendix, we further illustrate the fermion embeddings in this work.

After identifying the symmetries of canceling the leading quadratic divergence in the Higgs potential, we estimate how much the symmetries are expected to be explicitly broken at the relevant cutoff scale $\Lambda$. The leading breaking arises from the differential running, due to different top partners having different gauge charges, and there are more states in $\tilde{Q}_{L}$ than those in $Q_{L}$. Starting from the scale $\Lambda_{\not_{2}}$, which is the order of $m_{\tilde{X}}$ as defined in the Appendix, at which the differential running of different top partners and the top quark initially arise, the breaking at the scale $\Lambda$ could be estimated. Following the estimation in Ref. [15], we obtain

$$
\begin{aligned}
\Delta y^{2}= & y^{2}-\tilde{y}^{2} \\
\simeq & y^{2}\left(\frac{A_{1} g_{1}^{2}+3 A_{2} g_{2}^{2}}{16 \pi^{2}}-\frac{\tilde{A}_{1} g_{1}^{2}+3 \tilde{A}_{2} g_{2}^{2}+\tilde{A}_{3} g_{1}^{2}}{16 \pi^{2}}\right) \log \frac{\Lambda_{\not_{2}}}{\Lambda} \\
& +y^{2}\left(\frac{A_{3} g_{3}^{2}}{16 \pi^{2}}-\frac{\left(\tilde{A}_{4}+\tilde{A}_{5}\right) \tilde{g}_{3}^{2}}{16 \pi^{2}}\right) \log \frac{\Lambda_{\not_{2}}}{\Lambda}
\end{aligned}
$$

where $A_{1,2,3}$ and $\tilde{A}_{1,2,3,4,5}$ are $\mathcal{O}(1)$ unknown coefficients denoting the corrections of the gauge loops to the SM top quark and top partners; $g_{1,2}$ and $g_{3}, \tilde{g}_{3}$ denote the gauge couplings of the electroweak (EW) $U(1)_{Y}$, the EW $S U(2)_{L}$ group, the QCD $S U(3)_{c}$ color group, and its neutralnaturalness counterpart $S U(3)_{c}^{\prime}$, respectively. Note that this feature of explicitly breaking is universal in various twin Higgs and neutral-naturalness models; in the following we assume the breaking is small enough and can be neglected, i.e., the various coefficients satisfy $A_{1}=\tilde{A}_{1}+\tilde{A}_{3}, A_{2}=\tilde{A}_{2}$, and $A_{3}=\tilde{A}_{4}+\tilde{A_{5}}$ with $g_{3}(\Lambda)=\tilde{g}_{3}(\Lambda)$. Similar corrections from the Yukawa couplings exist as $\Delta y^{2} \sim \frac{y^{4}}{16 \pi^{2}} \log \frac{\Lambda_{Z_{2}}}{\Lambda}$. In order to precisely determine the Higgs potential, the corrections of the renormalization group running from the cutoff scale $\Lambda$ down to the EW scale, at which the Higgs mass is measured, are needed. We refer to Ref. [15] for detailed discussion.

\section{FULLY RADIATIVE HIGGS POTENTIAL}

Following Coleman-Weinberg [36], one obtains the oneloop Higgs potential. Instead of presenting lengthy expressions, we demonstrate the vacuum misalignment is obtained naturally with mass insertion method.

First, let us address quadratic divergence cancellation when additional symmetries, i.e., $S U(6)$ or $Z_{2}$ [defined as external to $S O(5)$ rotation] plus $S O(5)$ rotation, impose $y=\tilde{y}$. According to Fig. 1, the Higgs quadratic term from each diagram reads

$$
V(h) \sim \frac{y^{2} f^{2} N_{c} \Lambda^{2}}{16 \pi^{2}}\left(\frac{1}{2} s_{h}^{2}+\frac{1}{2} s_{h}^{2}+c_{h}^{2}\right),
$$

where $N_{c}=3$ and $\Lambda$ denotes the UV cutoff. The mass parameter $m_{\tilde{q}}$ is irrelevant to $\Lambda^{2}$. Even if the doublet 

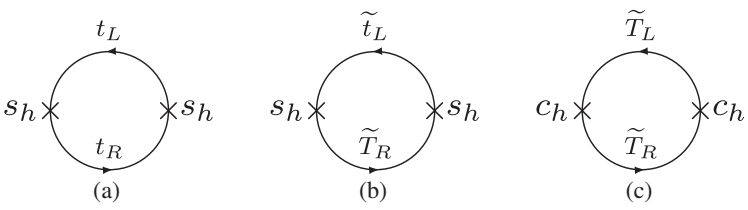

FIG. 1. Quadratic divergence cancellation between the top quark and the top partners using mass insertion. Each cross $(\times)$ denotes a Higgs insertion.

fermion is decoupled $\left(m_{\tilde{q}} \rightarrow \infty\right)$, the quadratic divergence can be canceled by only the singlet fermion.

Then we investigate the logarithmic divergence of the Higgs potential. We temporally neglect the contribution from the mass term in Eq. (5). According to Fig. 2, the logarithmic divergent part from diagrams (a)-(e) reads

$$
\begin{aligned}
V(h) & \sim \frac{y^{4} f^{4} N_{c} \log \Lambda^{2}}{16 \pi^{2}}\left(\frac{1}{4} s_{h}^{4}+\frac{1}{4} s_{h}^{4}+c_{h}^{4}+\frac{1}{2} s_{h}^{2} c_{h}^{2}+\frac{1}{2} s_{h}^{2} c_{h}^{2}\right) \\
& \sim \frac{y^{4} f^{4} N_{c} \log \Lambda^{2}}{16 \pi^{2}}\left(-s_{h}^{2}+\frac{1}{2} s_{h}^{4}\right) .
\end{aligned}
$$

The electroweak symmetry breaking is triggered by the $c_{h}^{4}$ term from the singlet fermion $\tilde{T}$ loop. Without contribution from the mass term, the electroweak scale is obtained to be $v=f$, which is too large to be compatible with data.

Finally, we include the log-dependent Higgs potential from the mass term $m_{\tilde{q}} \overline{\tilde{Q}}_{L} \tilde{Q}_{R}$. According to Fig. 2(f), it provides a positive $s_{h}^{2}$ contribution

$$
V(h) \sim \frac{y^{2} f^{2} N_{c} \log \Lambda^{2}}{16 \pi^{2}} m_{\tilde{q}}^{2} s_{h}^{2}
$$

Combined with the negative $s_{h}^{2}$ contribution in Eq. (11), we obtain the complete log-dependent Higgs potential

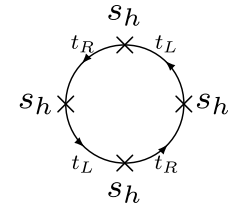

(a)

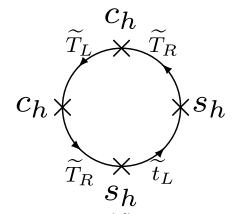

(d)

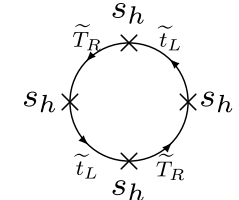

(b)

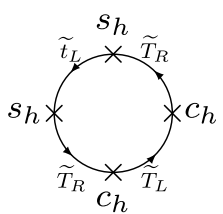

(e)

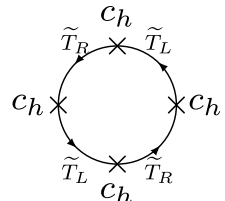

(c)

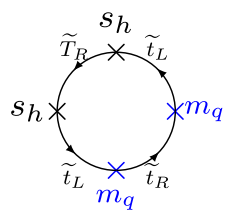

(f)
FIG. 2. The logarithmic divergent Higgs potential. Diagrams (a)-(e) denote the contribution induced by only the Yukawa terms in Eq. (5), and diagram (f) denotes the contribution from the doublet fermion mass term $m_{\tilde{q}} \tilde{\tilde{Q}}_{L} \tilde{Q}_{R}$.
$V(h) \sim \frac{y^{2} f^{2} N_{c} \log \Lambda^{2}}{16 \pi^{2}}\left[\left(m_{\tilde{q}}^{2}-y^{2} f^{2}\right) s_{h}^{2}+\frac{y^{2} f^{2}}{2} s_{h}^{4}\right]$.

The coefficient of the $s_{h}^{2}$ term can be much smaller than that of the $s_{h}^{4}$ term, due to the cancellation between the Yukawa terms, which mainly originates from the singlet contribution $c_{h}^{4}$ (and hence it leads to a negative $s_{h}^{2}$ term), and the doublet fermion mass term, which generates the positive $s_{h}^{2}$ term. As mentioned in the Introduction, unlike cancellation between two independent sectors, such as fermions and gauge bosons in twin Higgs models [30,31], we provide a novel way to suppress the $s_{h}^{2}$ relative to the $s_{h}^{4}$ term with only the top sector. In other models, usually additional shift-symmetry breaking sources are needed for realizing the suppression of the $s_{h}^{2}$ term, or the enhancement of the $s_{h}^{4}$ term.

As a result, we are able to obtain the vacuum misalignment without including a contribution from the bosonic sector. The vacuum misalignment is parametrized by

$$
\xi \equiv \frac{v^{2}}{f^{2}} \simeq 1-\frac{m_{\tilde{q}}^{2}}{y^{2} f^{2}}
$$

Since the top partner mass is naturally at the order of $m_{\tilde{q}} \sim y f, \xi \sim 0.1$ can be realized. In our numerical study, we further include the finite part of the Higgs potential, which does not change the general result depicted above.

The key ingredients in our setup are the doublet and singlet top partners, charged only under the electroweak gauge symmetry, but not the QCD gauge symmetry. The mixings between the SM top quark and the top partners are forbidden. These mixings are dangerous for the cancellation in the radiative Higgs potential shown in Figs. 1 and 2, because they could introduce extra terms (and thus more diagrams) with nontrivial Higgs dependence. Therefore, it is worthwhile pointing out that the cancellation in our model does not apply to minimal composite Higgs models with doublet and singlet top partners charged under the SM QCD, in which general mixing terms between all the fermions are allowed.

\section{ULTRAVIOLET REALIZATION}

Typically the PNGB Higgs can originate from new strong dynamics above the $\mathrm{TeV}$ scale, which introduces compositeness. The strong dynamics can effectively be described with the holographic framework [32]. Introducing composite fermions further gets rid of the $\log \Lambda^{2}$ dependence in the Higgs potential of Eq. (13), with the scale $\Lambda$ being interpreted as composite state masses. In MNNM, the gauge boson loops still encounter $\Lambda^{2}$ dependence. Introducing composite gauge bosons cancels both the $\Lambda^{2}$ and $\log \Lambda^{2}$ dependence. These render the full Higgs potential finite.

In the extra dimensional setup, the Higgs boson is identified as the zero mode of the fifth dimensional gauge 
field $A_{5}\left(x^{\mu}, z\right)$ [37,38]. The five-dimensional (5D) metric of $\mathrm{AdS}_{5}$ is $d s^{2}=\left(\frac{L}{z}\right)^{2}\left(\eta_{\mu \nu} d x^{\mu} d x^{\nu}-d z^{2}\right)$, where the UV and IR branes are localized at $z_{\mathrm{UV}}=L_{0}$ and $z_{\mathrm{IR}}=L_{1}$, respectively. The bulk gauge symmetry $S O(5) \times U(1)_{X} \times$ $S U(3)_{c} \times S U(3)_{c}^{\prime}$ is broken to $S O(4) \times U(1)_{X} \times S U(3)_{c} \times$ $S U(3)^{\prime}{ }_{c}^{\prime}$ on the IR brane, while is further reduced to $S U(2)_{L} \times U(1)_{Y} \times S U(3)_{c} \times S U(3)_{c}^{\prime}$ on the UV brane, with the hypercharge $Y=X+T_{R}^{3}$.

For the fermions in the SM sector, $q_{L}$ and $t_{R}$ are, respectively, identified as the zero modes of the bulk fields $\xi_{q}$ and $\xi_{t}$ with corresponding boundary conditions

$$
\begin{aligned}
& \xi_{q}=\left[\begin{array}{cr}
(2,2)_{L}^{q}=\left[\begin{array}{l}
q_{L}^{\prime}(-+) \\
q_{L}(++)
\end{array}\right] & (2,2)_{R}^{q}=\left[\begin{array}{l}
q_{R}^{\prime}(+-) \\
q_{R}(--)
\end{array}\right] \\
(1,1)_{L}^{q}(-+) & (1,1)_{R}^{q}(+-)
\end{array}\right],
\end{aligned}
$$

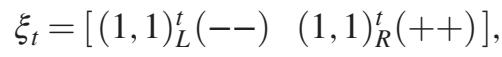

where $( \pm, \pm)$ denote the Neumann $(+)$ and Dirichlet $(-)$ boundary conditions (B.C.) on the UV and IR branes. Note that $\xi_{q}$ and $\xi_{t}$ are charged under the $S U(3)_{c}$ but neutral under the $S U(3)_{c}^{\prime}$. The above fermion assignment respects the $S O(5)$ symmetry on the IR brane, which renders the Higgs as the exact Goldstone. We add the IR-brane localized term

$$
\mathcal{L} \supset \frac{m}{g_{5}^{2}} \overline{(1,1)_{L}^{q}}(1,1)_{R}^{t}\left(z_{\mathrm{IR}}=L_{1}\right)+\text { H.c. },
$$

where $m$ is a dimensionless mass parameter and $g_{5}$ is a $5 \mathrm{D}$ gauge parameter with $\operatorname{Dim}\left[1 / g_{5}^{2}\right]=1$. This term explicitly breaks the $S O(5)$ symmetry on the IR brane and thus generates the top quark mass and the finite Higgs potential.

For the fermions in the hidden sector, the elementary fermion doublet $\tilde{q}_{R}$ is only localized on the UV brane, while the elementary fermion doublet $\tilde{q}_{L}$ and singlet $\tilde{T}_{L, R}$ are embedded in the bulk fermions $\xi_{\tilde{q}}$ and $\xi_{\tilde{T}}$, respectively, as follows:

$$
\begin{aligned}
& \xi_{\tilde{q}}=\left[\begin{array}{cr}
(2,2)_{L}^{\tilde{q}}=\left[\begin{array}{l}
\tilde{q}_{L}^{\prime}(-+) \\
\tilde{q}_{L}(++)
\end{array}\right] & (2,2)_{R}^{\tilde{q}}=\left[\begin{array}{l}
\tilde{q}_{R}^{\prime}(+-) \\
\tilde{q}_{R}(--)
\end{array}\right] \\
(1,1)_{L}^{\tilde{q}}(++) & (1,1)_{R}^{\tilde{q}}(--)
\end{array}\right],
\end{aligned}
$$

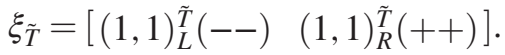

The bulk fermions $\xi_{\tilde{q}}$ and $\xi_{\tilde{T}}$ are charged under the $S U(3)_{c}^{\prime}$ while neutral under the $S U(3)_{c}$. Similarly, the IR-brane term, which breaks the fermionic $S O(5)$ symmetry on the IR brane, is

$$
\mathcal{L} \supset \frac{\tilde{m}}{g_{5}^{2}} \overline{(1,1)_{L}^{\tilde{q}}}(1,1)_{R}^{\tilde{T}}\left(z_{\mathrm{IR}}=L_{1}\right)+\text { H.c. }
$$

The dimensionless mass parameter $\tilde{m}$ induces an additional contribution to the finite Higgs potential and the hidden fermion masses. According to our UV brane assignment, we introduce the UV-brane localized mass term for the doublet fermion

$$
\mathcal{L} \supset-\frac{\tilde{m}_{q}}{g_{5}^{2}} \overline{\tilde{q}}_{R} \tilde{q}_{L}(++)\left(z_{\mathrm{UV}}=L_{0}\right)+\text { H.c. }
$$

which respects the gauge symmetry on the UV brane.

Following dimensional deconstruction [39], the above extra dimensional setup can effectively be described by multisite moose models [40,41]. The minimal moose setup is the two-site model based on the $S O(5)_{1} \times S O(5)_{2} / S O(5)_{V}$ coset $[42,43]$ with $S O(4)_{2}$ gauged on the 2-site. The $\Lambda^{2}$ dependence from the SM gauge bosons is canceled by the composite $\rho$ mesons introduced by gauging $\mathrm{SO}(4)_{2}$. This can be viewed as the extension of the $S O(5) / S O(4)$ coset in Sec. II with composite states. Under the paradigm of partial compositeness [44], composite partners $\Psi_{L, R}$ and their counterparts $\tilde{\Psi}_{L, R}$ in the color-neutral sector are introduced to mimic the Kaluza-Klein states in the holographic setup. The fermion assignment is shown as the moose diagram in Fig. 3. The link field $U$ corresponds to the Wilson line along the fifth dimension in the 5D model.

With the $S O(4)_{2}$ gauge symmetry on the 2-site, we can decompose the composite $\Psi(\tilde{\Psi})$ to $\Psi^{(4)}\left(\tilde{\Psi}^{(4)}\right)$ and $\Psi^{(1)}$ $\left(\tilde{\Psi}^{(1)}\right)$. The fermionic Lagrangian reads

$$
\begin{aligned}
\mathcal{L}= & y f \bar{Q}_{L} U \Psi_{R}-M \bar{\Psi}_{L} \Psi_{R}-m \bar{\Psi}_{L}^{(1)} t_{R} \\
& +\tilde{y} f \overline{\tilde{Q}}_{L} U \tilde{\Psi}_{R}-\tilde{M} \tilde{\tilde{\Psi}}_{L} \tilde{\Psi}_{R}-\tilde{m} \tilde{\tilde{\Psi}}_{L}^{(1)} \tilde{T}_{R} \\
& -\tilde{m}_{q} \overline{\tilde{Q}}_{L} \tilde{Q}_{R}+\text { H.c. },
\end{aligned}
$$

where mass splittings of $\Psi_{4,1}\left(\tilde{\Psi}_{4,1}\right)$ are assumed to be zero. Since $Q_{L}$ and $\tilde{Q}_{L, R}$ form incomplete $S O(5)$ multiplets, $S O(5)_{1}$ is explicitly broken. Without introducing mass terms $m \bar{\Psi}_{L}^{(1)} t_{R}$ and $\tilde{m} \overline{\tilde{\Psi}}_{L}^{(1)} \tilde{T}_{R}$, the $S O(5)_{2}$ remains unbroken, then the Higgs is an exact Goldstone. The nonvanishing Higgs potential can only exist when both $S O(5)_{1}$ and $S O(5)_{2}$ are explicitly broken, which is referred to as collective symmetry breaking $[42,45]$.

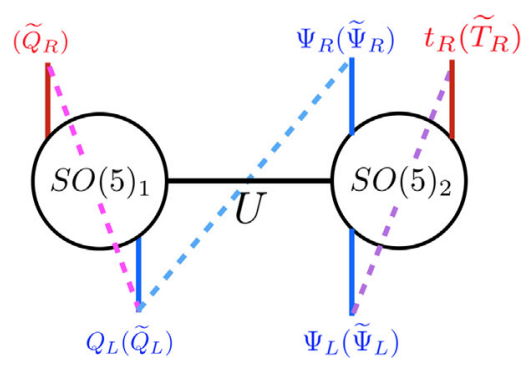

FIG. 3. The two-site moose diagram and fermion assignment for composite neutral naturalness. Each circle denotes a site and the link in between denotes the Goldstone matrix. 
In both frameworks, one can integrate out the bulk dynamics or the composite fermions $\Psi$ and $\tilde{\Psi}$ in Eq. (21) and match to the holographic effective Lagrangian $[26,38,46,47]$. Then the finite Higgs potential is obtained

$$
\begin{aligned}
V(h) \simeq & -\frac{3\left(a y^{2} f^{2} m^{2}-\tilde{a} \tilde{y}^{2} f^{2} \tilde{m}^{2}\right)}{16 \pi^{2}} s_{h}^{2} \\
& +\frac{3\left(b y^{4} f^{4}+\tilde{b} \tilde{y}^{4} f^{4}\right)}{16 \pi^{2}} s_{h}^{4},
\end{aligned}
$$

where parameters $a, \tilde{a}, b, \tilde{b}$ depend on the bulk masses and mixings in the holographic model, or the parameters in Eq. (21) for the composite model. The details of tedious calculation will be presented in a forthcoming publication (see also Supplemental Materials [48]). The $s_{h}^{2}$ term contains two opposite contributions from the SM and the hidden sector, which suppress its relative magnitude compared to the $s_{h}^{4}$ term and realize vacuum misalignment. The corresponding fine-tuning level of our model is similar to the one of other composite Higgs models [29,49].

\section{MODEL IMPLICATION AND CONCLUSION}

This MNNM setup solves the little hierarchy problem with the minimal hidden sector. The hidden sector contains dark $S U(3)_{c}^{\prime}$ color, under which new electroweak singlet and doublet fermions are charged. These fermions are responsible for generating the radiative Higgs potential with their masses around scale $f$. Since the dark color group is confined at around $\mathrm{GeV}$ scale and no new fermions are lighter than that, the heavy fermions exhibit quirk behavior [11,50] and form macroscopic bound states [51]. Depending on the electroweak charges of these fermions, the top partners can be neutral or charged particles, i.e., $Q=0,2 / 3,1$, etc. Discovering these exotic bound states forming from quirks is one of the smoking-gun signatures at colliders. For charged top partners, they can be produced through the Drell-Yan process and then form bound states [21,52-54]. For neutral top partners, it is more promising to detect them at the Large Hadron Collider (LHC) with the possible displaced vertex signature $[6,55,56]$ from Higgs exotic decay. Specifically in MNNM, there is at least one charged component in the doublet top partner, thus this charged component could yield a benchmark quirky signature. In order to fully verify the doublet-singlet spectrum of the top partners at colliders, one expects additional correlated quirky signatures or displaced vertices, yielded by the other component of the doublet and the singlet. The correlation between the signals of the doublet and the singlet is a natural consequence of our fermion embedding, as all the masses and couplings of the top partners are constrained if they are required to give rise to the small vacuum misalignment angle. On top of the minimal setup, there exist colored Kaluza-Klein states (or, equivalently, composite fermions) in the holographic (composite Higgs) UV completion. Similar to the holographic/composite completion to the twin Higgs setup [12-14], these UV heavy states are typically at the order of 3-10 $\mathrm{TeV}$ and thus not too far beyond the reach of the LHC. It would be fascinating if any of the above signals are actually discovered.

Current searches at the LHC put constraints on the model parameters $f$ and $m_{\tilde{q}}$, which determine the low energy spectrum and couplings in MNNM. Since the Higgs boson is a PNGB connecting the SM and the hidden sector, the tightest constraint is from Higgs coupling measurements [57-59]. We perform a global analysis on the Higgs nonlinearity parameter $\xi=v^{2} / f^{2}$ and new fermion mass parameter $m_{\tilde{q}}$ using the latest Higgs data encoded in the program Lilith [60]. Figure 4 reads $\xi<0.1(0.2)$ at $1 \sigma(2 \sigma)$ confidence level. We also consider constraints on vectorlike top partners from electroweak precision tests, using the oblique parameters $\mathrm{S}, \mathrm{T}[61,62]$. The gray shaded region in Fig. 4 shows the dominated constraint from the $\mathrm{T}$ parameter on singlet and doublet fermion masses and mixing [63,64]. To obtain the correct vacuum misalignment and the $125 \mathrm{GeV}$ Higgs mass, model parameters need to be within the colored region in Fig. 4, with $\Lambda \subset[3 \mathrm{TeV}, 10 \mathrm{TeV}]$ and $y \subset[0.86,0.98]$ corresponding to the running top quark mass at $\mathrm{TeV}$ scale. The strong correlation between $\xi$ and $m_{\tilde{q}}$ shows the cancellation of singlet and doublet top partner contributions on the Higgs quadratic term, and thus determines the fine-tuning level of the model. According to Eq. (1), we define the fine-tuning level [29] as

$$
\Delta \equiv \frac{\left.\gamma\right|_{m_{\tilde{q}}=0}}{\gamma_{0}} \cdot \frac{\left.\beta\right|_{m_{\tilde{q}}=0}}{\beta_{0}} \sim \mathcal{O}(10),
$$

where $\gamma_{0}$ and $\beta_{0}$ are the correct values for obtaining vacuum misalignment and the Higgs mass. As shown in Fig. 4, the

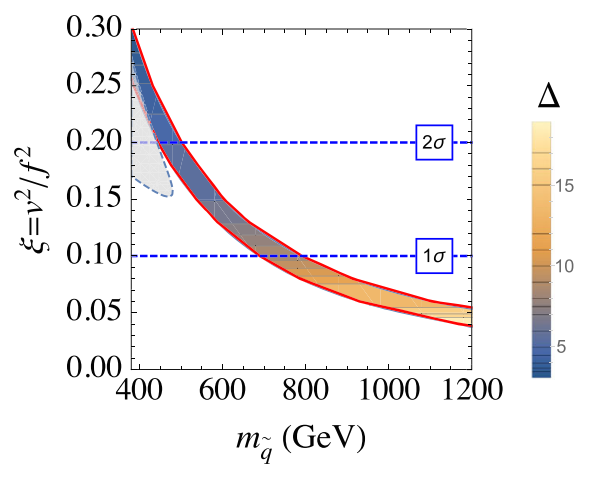

FIG. 4. The colored region denotes the viable parameter space on $\left(\xi, m_{\tilde{q}}\right)$, in which vacuum misalignment and the $125 \mathrm{GeV}$ Higgs mass are obtained, with electric charge of the top partners unspecified. The color bar on the right shows the fine-tuning level. The blue dashed lines denote the $1 \sigma, 2 \sigma$ contours allowed by Higgs data, assuming electric charge $Q=1$ for the hidden tops. The gray shaded region denotes the $\mathrm{T}$ parameter constraints to the top partners. 
smaller $\xi$, the larger $m_{\tilde{q}}$, and severer fine-tuning. The SM is recovered as $m_{\tilde{q}} \rightarrow \infty$ and $\xi \rightarrow 0$.

The hidden sector can naturally accommodate dark matter candidates. If the top partners are charge neutral, they form the lightest dark baryon which serves as dark matter in the asymmetric dark matter scenario [65-69]. In this minimal setup, it is not easy to identify the dark baryon or meson as WIMP dark matter because of strong interactions in the dark color sector. One needs to introduce either light dark-colored fermions to have strongly interacting massive particle dark matter [70] or leptons in the hidden sector to have singlet-doublet fermion dark matter $[71,72]$.

Overall, we lay out the basic setup of a minimal neutral naturalness model and its UV extension with emphasis on generating the realistic Higgs potential. The mechanism for obtaining vacuum misalignment through fermion-fermion cancellation is novel, and it can possibly be generalized to other neutral naturalness and composite Higgs models. This setup contains very rich phenomenology which cannot be expanded in this paper, such as dark hadron spectra, collider signatures, cosmological implications, heavy composite particles, etc. We leave these directions for future explorations.

\section{ACKNOWLEDGMENTS}

J. H. Y. is supported by the National Science Foundation of China under Grant No. 11875003 and the Chinese Academy of Sciences (CAS) Hundred-Talent Program. L.X.X. and S.H.Z. are supported in part by the National Science Foundation of China under Grants No. 11635001, No. 11875072.

\section{APPENDIX: MORE COMMENTS ON FERMION EMBEDDINGS}

In this appendix, we would like to point out the novelty of the fermion embeddings proposed in this work, by comparing our model to the models with trigonometric parity, e.g., the model with the coset $S O(6) / S O(5)$ [or equivalently $S U(3) / S U(2)$ without custodial symmetry] $[15,16]$. The main differences are as follows.

(i) To cancel the quadratic divergence in the Higgs mass term, the fermion embedding in the $S O(5) / S O(4)$ coset is quite different from the one in the $S O(6) / S O(5)$ [and $S U(3) / S U(2)$ ] coset. To be concrete, the fermion embedding in the $S O(6) /$ $S O(5)$ setup is

$$
\Psi_{Q_{L}}=\frac{1}{\sqrt{2}}\left(\begin{array}{c}
b_{L} \\
-i b_{L} \\
t_{L} \\
i t_{L} \\
0 \\
0
\end{array}\right), \quad \Psi_{\tilde{t}_{L}}=\frac{1}{\sqrt{2}}\left(\begin{array}{c}
0 \\
0 \\
0 \\
0 \\
\tilde{t}_{L} \\
i \tilde{t}_{L}
\end{array}\right) .
$$

The Yukawa Lagrangian is

$$
y_{t} \bar{\Psi}_{Q_{L}} \Sigma t_{R}+\tilde{y}_{t} \bar{\Psi}_{\tilde{t}_{L}} \Sigma \tilde{t}_{R}+\text { H.c. }
$$

We see that only the fourth component of the $\Psi_{Q_{L}}$, and the sixth component of the $\Psi_{\tilde{t}_{L}}$ have the $s_{h}$ and $c_{h}$ dependence in the Yukawa term, respectively. Furthermore, an internal $Z_{2}$ trigonometric parity is obtained by the combination of the $\mathrm{SO}(2)$ rotation by angle $\pi / 2$ with the Higgs parity transformation (outer automorphism), which exchanges the 4th and 6th rows inside one fermion multiplet. However, as stated in Ref. [16], to extend the $Z_{2}$ trigonometric parity to the Yukawa couplings, an additional external $Z_{2}$ parity needs to be introduced to relate two different fermion multiplets. Taken $y_{t}=\tilde{y}_{t}$, the Yukawa term satisfies the trigonometric parity with an external $Z_{2}$ parity

$t_{L} \leftrightarrow \tilde{t}_{L}, \quad t_{R} \leftrightarrow \tilde{t}_{R}, \quad \sin \frac{h}{f} \leftrightarrow \cos \frac{h}{f}$,

and quadratic divergence cancellation is realized as

$$
V(h) \supset \frac{1}{2} y_{t}^{2} \Lambda^{2}\left(\sin ^{2} \frac{h}{f}+\cos ^{2} \frac{h}{f}\right) .
$$

On the other hand, in the $S O(5) / S O(4)$ setup, if we take the similar fermion embedding

$\Psi_{Q_{L}}=\frac{1}{\sqrt{2}}\left(\begin{array}{c}b_{L} \\ -i b_{L} \\ t_{L} \\ i t_{L} \\ 0\end{array}\right), \quad \Psi_{\tilde{t}_{L}}=\left(\begin{array}{c}0 \\ 0 \\ 0 \\ 0 \\ \tilde{t}_{L}\end{array}\right)$

The same Yukawa term cannot ensure the quadratic divergence cancellation when $y_{t}=\tilde{y}_{t}$. Even the same outer automorphism exists in $S O(5)$, it cannot be used to related the top quark and the top partner. The fourth component of the $\Psi_{Q_{L}}\left(s_{h}\right.$ dependence), and the fifth component of the $\Psi_{\tilde{t}_{L}}\left(c_{h}\right.$ dependence) would induce

$$
\begin{aligned}
V(h) & \supset \frac{1}{2} y_{t}^{2} \Lambda^{2}\left(\sin ^{2} \frac{h}{f}+2 \cos ^{2} \frac{h}{f}\right) \\
& \sim \frac{1}{2} y_{t}^{2} \Lambda^{2} \cos ^{2} \frac{h}{f},
\end{aligned}
$$

due to the extra factor of $\sqrt{2}$.

(ii) Therefore, additional degree of freedom with the $s_{h}$ dependence (which is the fermion with $S U(2)_{L}$ doublet quantum number) should be introduced 
and be implemented in the fermion embedding as in Eq. (4). There is a symmetry argument which determines the above embeddings. First of all, let us embed all the elementary fields into the fundamental representation of $S O(5)$ as

$$
\begin{array}{ll}
Q_{L}=\frac{1}{\sqrt{2}}\left(\begin{array}{c}
b_{L} \\
-i b_{L} \\
t_{L} \\
i t_{L} \\
0
\end{array}\right), & \\
\tilde{Q}_{L 1}=\frac{1}{\sqrt{2}}\left(\begin{array}{c}
\tilde{b}_{L} \\
-i \tilde{b}_{L} \\
\tilde{t}_{L} \\
i \tilde{t}_{L} \\
0
\end{array}\right), \quad \tilde{Q}_{L 2}=\left(\begin{array}{c}
0 \\
0 \\
0 \\
0 \\
\tilde{T}_{L}
\end{array}\right),
\end{array}
$$

while $\tilde{Q}_{L}$ is defined as

$$
\tilde{Q}_{L}=\alpha \tilde{Q}_{L 1}+\beta \tilde{Q}_{L 2} .
$$

Note that, up to now, the coefficients $\alpha$ and $\beta$ are in general arbitrary. Lack of a $S O(5)$-internal symmetry would leave us the arbitrariness the ratio of $\alpha$ and $\beta$ in the Yukawa coupling. We argue $\alpha / \beta=1$, as needed for neutral naturalness cancellation, is due to an internal $S O(5)$ rotation, if $\tilde{Q}_{L}$ is completed into a full $S O(5)$ 5-plet. This could be realized by introducing another $S U(2)_{L}$ doublet spurion field $\tilde{X}_{L, R}$ with hypercharge $X+1 / 2$, but only charged under the hidden color group (neutral under the SM QCD color). One can embed $\tilde{X}_{L}$ into fundamental representation of $S O(5)$ global symmetry in a similar way as $\tilde{q}_{L}$,

$$
\tilde{Q}_{L 3}=\frac{1}{\sqrt{2}}\left(\begin{array}{c}
-\tilde{X}_{u L} \\
-i \tilde{X}_{u L} \\
\tilde{X}_{d L} \\
-i \tilde{X}_{d L} \\
0
\end{array}\right)
$$

where $\tilde{X}_{u}$ and $\tilde{X}_{d}$ are the upper and lower components of the doublet $\tilde{X}$, respectively. The full $S O(5)$ symmetric fundamental is defined as

$$
\tilde{Q}_{L}^{\prime}=\alpha \tilde{Q}_{L 1}+\beta \tilde{Q}_{L 2}+\gamma \tilde{Q}_{L 3},
$$

with the coefficients $\alpha=\beta=\gamma$. With the full $S O(5)$-symmetric fundamental $\tilde{Q}_{L}^{\prime}$ we rewrite the Yukawa sector as

$$
\begin{aligned}
\mathcal{L}_{\text {top }}= & -y \bar{Q}_{L} \Sigma t_{R}-\tilde{y} \overline{\tilde{Q}}_{L}^{\prime} \Sigma \tilde{T}_{R} \\
& -m_{\tilde{q}} \overline{\tilde{q}}_{L} \tilde{q}_{R}-m_{\tilde{X}} \overline{\tilde{X}}_{L} \tilde{X}_{R}+\text { H.c. }
\end{aligned}
$$

Here the soft mass terms for the $\tilde{q}$ and $\tilde{X}$ fields are introduced, which softly break the internal global $S O(5)$ symmetry, but do not affect the quadratic divergence cancellation. The soft term of $m_{\tilde{q}}$ is utilized to realize vacuum misalignment as shown in the manuscript, while the term of $m_{\tilde{X}}$ is used to decouple the doublet state $\tilde{X}$ [and thus softly breaks the $S O(5)$ rotation symmetry]. In other words, one can think $m_{\tilde{X}}$ as a $S O(5)$-breaking spurion, and in the limit $m_{\tilde{X}} \rightarrow \infty$ we have the original theory.

Let us elaborate the symmetry in the above Lagrangian. First, we have the internal $\mathrm{SO}(5)$ rotation relating $\tilde{Q}_{L 1,2,3}$ inside the full $S O(5)$ fundamental $\tilde{Q}^{\prime}$, which determine the coefficients to be $\alpha=\beta=\gamma=1$; second, we have the $Z_{2}$ parity [SO(5)-external] defined as $Q_{L} \leftrightarrow \tilde{Q}_{L}^{\prime}, t_{R} \leftrightarrow \tilde{T}_{R}$, which determines $y=\tilde{y}$. Likewise one can also think $Q_{L}$ in the SM sector as a full $S O(5)$ fundamental, in which the unphysical components are decoupled by soft mass terms, such that the $Z_{2}$ parity can be realized at the level of full $S O(5)$ multiplets. The Yukawa terms respect these symmetries, such that the one-loop Higgs potential is insensitive to quadratic divergence, as shown in Fig. 1.

The quadratic divergence cancellation does not rely on the soft mass terms with the masses being $m_{\tilde{q}}$ and $m_{\tilde{X}}$, respectively. We note that this softly-broken $S O(5)$ rotation could lead to quadratic divergence beyond one-loop level (in twin Higgs models, since the $Z_{2}$ twin parity is not exact, the same problem arises), whose magnitude however is small enough so that we can neglect it. Beyond the $10 \mathrm{TeV}$, once the low-energy effective model is UV completed, e.g., in the holographic approach, the Higgs potential becomes fully calculable and the problem could be resolved.

In the holographic setup, apart from the $Z_{2}$ parity between two $S O(5)$ multiplets, we similarly require the brane-localized kinetic terms respect the internal $S O(5)$ rotation, so that the corresponding low-energy neutral-naturalness cancellation is not spoiled. 
[1] M. Aaboud et al. (ATLAS Collaboration), J. High Energy Phys. 12 (2017) 085.

[2] M. Aaboud et al. (ATLAS Collaboration), Phys. Rev. Lett. 121, 211801 (2018).

[3] A. M. Sirunyan et al. (CMS Collaboration), Eur. Phys. J. C 77, 327 (2017).

[4] V. Khachatryan et al. (CMS Collaboration), Phys. Rev. D 96, 012004 (2017).

[5] R. Barbieri and A. Strumia, in 4th Rencontres du Vietnam: Physics at Extreme Energies (Particle Physics and Astrophysics) Hanoi, Vietnam (2000).

[6] N. Craig, A. Katz, M. Strassler, and R. Sundrum, J. High Energy Phys. 07 (2015) 105.

[7] N. Craig, S. Knapen, and P. Longhi, Phys. Rev. Lett. 114, 061803 (2015).

[8] Z. Chacko, H.-S. Goh, and R. Harnik, Phys. Rev. Lett. 96, 231802 (2006).

[9] G. Burdman, Z. Chacko, H.-S. Goh, and R. Harnik, J. High Energy Phys. 02 (2007) 009.

[10] D. Poland and J. Thaler, J. High Energy Phys. 11 (2008) 083.

[11] H. Cai, H.-C. Cheng, and J. Terning, J. High Energy Phys. 05 (2009) 045.

[12] M. Geller and O. Telem, Phys. Rev. Lett. 114, 191801 (2015).

[13] R. Barbieri, D. Greco, R. Rattazzi, and A. Wulzer, J. High Energy Phys. 08 (2015) 161.

[14] M. Low, A. Tesi, and L.-T. Wang, Phys. Rev. D 91, 095012 (2015).

[15] J. Serra and R. Torre, Phys. Rev. D 97, 035017 (2018).

[16] C. Csaki, T. Ma, and J. Shu, Phys. Rev. Lett. 121, 231801 (2018).

[17] B. M. Dillon, Phys. Rev. D 99, 115008 (2019).

[18] T. Cohen, N. Craig, G. F. Giudice, and M. Mccullough, J. High Energy Phys. 05 (2018) 091.

[19] H.-C. Cheng, L. Li, E. Salvioni, and C. B. Verhaaren, J. High Energy Phys. 05 (2018) 057.

[20] H.-C. Cheng, S. Jung, E. Salvioni, and Y. Tsai, J. High Energy Phys. 03 (2016) 074.

[21] Z. Chacko, D. Curtin, and C. B. Verhaaren, Phys. Rev. D 94, 011504 (2016).

[22] N. Craig, S. Koren, and T. Trott, J. High Energy Phys. 05 (2017) 038.

[23] Z. Chacko, N. Craig, P. J. Fox, and R. Harnik, J. High Energy Phys. 07 (2017) 023.

[24] C. Csaki, E. Kuflik, and S. Lombardo, Phys. Rev. D 96, 055013 (2017).

[25] Z. Chacko, D. Curtin, M. Geller, and Y. Tsai, J. High Energy Phys. 09 (2018) 163.

[26] K. Agashe, R. Contino, and A. Pomarol, Nucl. Phys. B719, 165 (2005).

[27] D. B. Kaplan and H. Georgi, Phys. Lett. 136B, 183 (1984).

[28] D. Marzocca, M. Serone, and J. Shu, J. High Energy Phys. 08 (2012) 013.

[29] B. Bellazzini, C. Csaki, and J. Serra, Eur. Phys. J. C 74, 2766 (2014).

[30] J.-H. Yu, Phys. Rev. D 94, 111704 (2016).

[31] J.-H. Yu, J. High Energy Phys. 12 (2016) 143.

[32] N. Arkani-Hamed, M. Porrati, and L. Randall, J. High Energy Phys. 08 (2001) 017.
[33] R. Contino, in Physics of the Large and the Small, TASI 09, Proceedings of the Theoretical Advanced Study Institute in Elementary Particle Physics, Boulder, Colorado, 2009 (World Scientific Publishing Co., Singapore, 2011), pp. 235-306.

[34] G. Panico and A. Wulzer, Lect. Notes Phys. 913, 1 (2016).

[35] C. Csaki, T. Ma, and J. Shu, Phys. Rev. Lett. 119, 131803 (2017).

[36] S. R. Coleman and E. J. Weinberg, Phys. Rev. D 7, 1888 (1973).

[37] N. Arkani-Hamed, A. G. Cohen, and H. Georgi, Phys. Lett. B 513, 232 (2001).

[38] R. Contino, Y. Nomura, and A. Pomarol, Nucl. Phys. B671, 148 (2003).

[39] N. Arkani-Hamed, A. G. Cohen, and H. Georgi, Phys. Rev. Lett. 86, 4757 (2001).

[40] H. Georgi, Nucl. Phys. B266, 274 (1986).

[41] H.-C. Cheng, J. Thaler, and L.-T. Wang, J. High Energy Phys. 09 (2006) 003.

[42] R. Foadi, J. T. Laverty, C. R. Schmidt, and J.-H. Yu, J. High Energy Phys. 06 (2010) 026.

[43] G. Panico and A. Wulzer, J. High Energy Phys. 09 (2011) 135.

[44] D. B. Kaplan, Nucl. Phys. B365, 259 (1991).

[45] N. Arkani-Hamed, A. G. Cohen, E. Katz, and A. E. Nelson, J. High Energy Phys. 07 (2002) 034.

[46] R. Contino and A. Pomarol, J. High Energy Phys. 11 (2004) 058.

[47] M. Serone, New J. Phys. 12, 075013 (2010).

[48] See Supplemental Material at http://link.aps.org/ supplemental/10.1103/PhysRevD.101.095014 for the radiative generated finite Higgs potential in the holographic setup.

[49] G. Panico, M. Redi, A. Tesi, and A. Wulzer, J. High Energy Phys. 03 (2013) 051.

[50] G. Burdman, Z. Chacko, H.-S. Goh, R. Harnik, and C. A. Krenke, Phys. Rev. D 78, 075028 (2008).

[51] J. Kang and M. A. Luty, J. High Energy Phys. 11 (2009) 065.

[52] R. Harnik, G. D. Kribs, and A. Martin, Phys. Rev. D 84, 035029 (2011).

[53] S. Knapen, H. K. Lou, M. Papucci, and J. Setford, Phys. Rev. D 96, 115015 (2017).

[54] M. Farina and M. Low, Phys. Rev. Lett. 119, 111801 (2017).

[55] J. Liu, Z. Liu, and L.-T. Wang, Phys. Rev. Lett. 122, 131801 (2019).

[56] D. Curtin et al., Rep. Prog. Phys. 82, 116201 (2019).

[57] G. Aad et al. (ATLAS and CMS Collaborations), Phys. Rev. Lett. 114, 191803 (2015).

[58] G. Aad et al. (ATLAS and CMS Collaborations), J. High Energy Phys. 08 (2016) 045.

[59] G. Aad et al. (ATLAS Collaboration), Phys. Rev. D 101, 012002 (2020).

[60] J. Bernon and B. Dumont, Eur. Phys. J. C 75, 440 (2015).

[61] M. E. Peskin and T. Takeuchi, Phys. Rev. D 46, 381 (1992).

[62] M. Baak, M. Goebel, J. Haller, A. Hoecker, D. Kennedy, R. Kogler, K. Moenig, M. Schott, and J. Stelzer, Eur. Phys. J. C 72, 2205 (2012).

[63] L. Lavoura and J. P. Silva, Phys. Rev. D 47, 2046 (1993). 
[64] G. Cynolter and E. Lendvai, Eur. Phys. J. C 58, 463 (2008).

[65] D. E. Kaplan, M. A. Luty, and K. M. Zurek, Phys. Rev. D 79, 115016 (2009).

[66] K. M. Zurek, Phys. Rep. 537, 91 (2014).

[67] G. D. Kribs, T. S. Roy, J. Terning, and K. M. Zurek, Phys. Rev. D 81, 095001 (2010).

[68] M. Farina, J. Cosmol. Astropart. Phys. 11 (2015) 017.
[69] I. Garcia Garcia, R. Lasenby, and J. March-Russell, Phys. Rev. Lett. 115, 121801 (2015).

[70] Y. Hochberg, E. Kuflik, T. Volansky, and J. G. Wacker, Phys. Rev. Lett. 113, 171301 (2014).

[71] C. E. Yaguna, Phys. Rev. D 92, 115002 (2015).

[72] I. Garcia Garcia, R. Lasenby, and J. March-Russell, Phys. Rev. D 92, 055034 (2015). 